\title{
Applications of a Noninvasive Respiratory Volume Monitor for Critical Care Medicine
}

\author{
Joseph J Schlesinger MD
}

\begin{abstract}
Respiratory volume monitoring (RVM) has been developed to noninvasively measure minute ventilation $\left(\dot{\mathrm{V}}_{\mathrm{E}}\right)$, tidal volume, and breathing frequency and to display real-time respiratory curves in nonintubated patients. Although RVM was originally developed for post-anesthesia and monitored anesthesia care, we describe 3 applications for this monitor in an otherwise austere setting at a missionary hospital in Kijabe, Kenya. Applications of RVM can be utilized in any ICU in a developing or developed country. Key words: respiratory volume monitoring; minute ventilation; respiratory physiology; critical care medicine. [Respir Care 2015;60(5):e97-e100. (C) 2015 Daedalus Enterprises]
\end{abstract}

\section{Introduction}

Respiratory volume monitoring (RVM) has recently become available to noninvasively measure minute ventilation $\left(\dot{\mathrm{V}}_{\mathrm{E}}\right)$, tidal volume $\left(\mathrm{V}_{\mathrm{T}}\right)$, and breathing frequency and to display real-time respiratory curves in nonintubated patients. Continuous real-time measurements of $\dot{V}_{E}, V_{T}$, and breathing frequency can provide point-of-care respiratory assessment and initiate timely interventions. An impedance-based RVM device (ExSpiron 1Xi, Respiratory Motion, Waltham, Massachusetts) (Fig. 1) that uses an electrode PadSet placed on the thorax was cleared by the FDA and demonstrated to provide accurate measurements of $\dot{\mathrm{V}}_{\mathrm{E}}, \mathrm{V}_{\mathrm{T}}$, and breathing frequency in nonintubated subjects. ${ }^{1,2}$ Previous research identified a $\dot{\mathrm{V}}_{\mathrm{E}}$ risk stratification protocol to assist with opioid dosing using a predicted $\dot{V}_{\mathrm{E}}$ based on ideal body weight to indicate when patients might have a $\dot{\mathrm{V}}_{\mathrm{E}}$ that could be considered at risk $\left(\dot{\mathrm{V}}_{\mathrm{E}}<80 \%\right.$ predicted $\left.\dot{\mathrm{V}}_{\mathrm{E}}\right)$ or unsafe $\left(\dot{\mathrm{V}}_{\mathrm{E}}<40 \%\right.$ predicted $\left.\dot{\mathrm{V}}_{\mathrm{E}}\right) .^{3}$ Additional stud-

Dr Schlesinger is affiliated with the Division of Critical Care Medicine, Department of Anesthesiology, Vanderbilt University Medical Center, Nashville, Tennessee and the Division of Critical Care Medicine, Department of Anesthesiology, University of Nairobi, Nairobi, Kenya.

Dr Schlesinger has disclosed no conflicts of interest.

Correspondence: Joseph J Schlesinger MD, Vanderbilt University Medical Center, 1211 21st Avenue South, Medical Arts Building 526, Nashville, TN 37212. E-mail: joseph.j.schlesinger@vanderbilt.edu.

DOI: $10.4187 /$ respcare. 03744

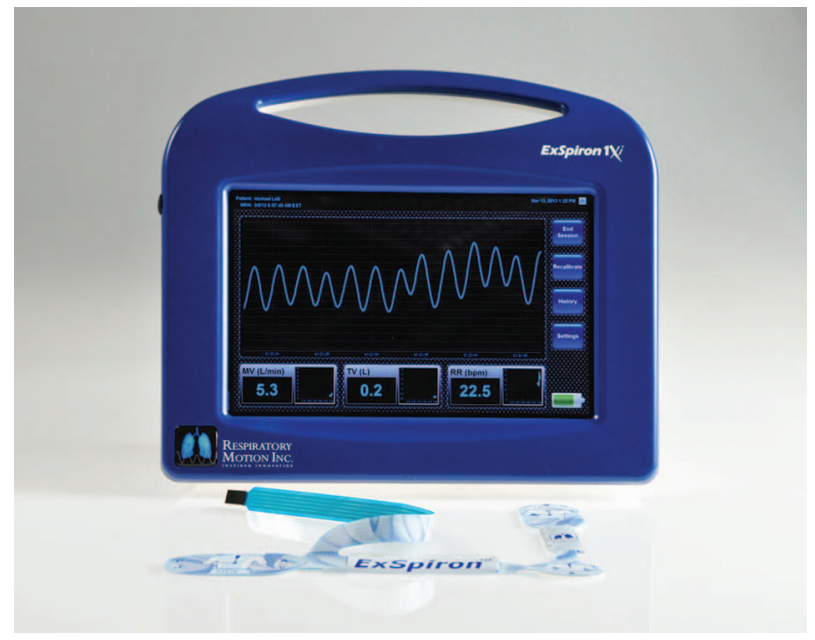

Fig. 1. The ExSpiron 1Xi respiratory volume monitoring device. Courtesy Respiratory Motion.

ies have demonstrated that subjects who receive opioids when their $\dot{\mathrm{V}}_{\mathrm{E}}$ is $<80 \%$ predicted $\dot{\mathrm{V}}_{\mathrm{E}}$ can have a marked decrease in $\dot{\mathrm{V}}_{\mathrm{E}}$ to $<40 \%$ predicted $\dot{\mathrm{V}}_{\mathrm{E}}{ }^{4,5}$ Because the device is relatively new, its average life span has not been established. However, the device is rugged and can receive remote software updates, and the company provides aroundthe-clock technical support. The only recurring cost is the inexpensive adhesive PadSet.

The following cases describe the use of RVM to help guide clinical decision making and educate health-care providers to improve patient care and safety. The responsible institutional review board gave permission to publish 
this report because attempts to contact the patients and families in Kenya were not successful.

\section{Case Reports}

\section{Case 1}

A 24-y-old Kenyan woman presented to Casualty (emergency department) with a left-sided facial droop and leftsided hemiplegia. Because of a concern for airway protection, she was intubated and admitted to the ICU. After extubation, she was unable to adequately clear her oropharyngeal secretions, became tachypneic, and was reintubated. Because she failed extubation, the surgical service was consulted regarding tracheotomy, which was performed with no complications. ${ }^{6}$ The patient was placed on pressure support spontaneous ventilation (Puritan Bennett 840 ventilator, Covidien, Mansfield, Massachusetts), and her respiratory status stabilized. However, the need for beds in the ICU made it necessary to quickly wean the patient to blow-by oxygen via a tracheostomy collar so that she could be sent to the step-down high-dependence unit (HDU). Because there was insufficient time for tracheostomy collar trials, we decided to use RVM to guide in weaning from mechanical ventilation.

For this patient, the predicted $\dot{\mathrm{V}}_{\mathrm{E}}$ based on ideal body weight was calculated as $5.2 \mathrm{~L} / \mathrm{min}$. RVM was initiated with a $\dot{V}_{\mathrm{E}}$ marker set at $80 \%$ predicted $\dot{\mathrm{V}}_{\mathrm{E}}(4.16 \mathrm{~L} / \mathrm{min})$ and the low $\dot{\mathrm{V}}_{\mathrm{E}}$ alarm set at $40 \%$ predicted $\dot{\mathrm{V}}_{\mathrm{E}}(2.08 \mathrm{~L} / \mathrm{min})$. The $\mathrm{F}_{\mathrm{IO}_{2}}$ and PEEP were weaned based on the peripheral capillary oxygen saturation $\left(\mathrm{S}_{\mathrm{pO}_{2}}\right)$ of a pulse oximeter because there was no capability to assess arterial blood gases. The amount of pressure support was weaned on the ventilator by $2 \mathrm{~cm} \mathrm{H}_{2} \mathrm{O}$ every 20 min as long as the $\dot{\mathrm{V}}_{\mathrm{E}}$ was $>40 \%$ predicted $\dot{\mathrm{V}}_{\mathrm{E}}$ because capnography was not available and the $\mathrm{V}_{\mathrm{T}}$ display on the ventilator available for the patient was not functioning.

The patient was successfully weaned from ventilatory support on postoperative day 0 after the tracheotomy and sent to the HDU within $3 \mathrm{~h}$ of arriving in the ICU after her operation. The noninvasive $\dot{V}_{\mathrm{E}}$ monitor allowed fast ventilator weaning by less experienced care providers in the absence of invasive and expensive laboratory work and equipment. The patient did well in the HDU and did not require readmission to the ICU. The RVM electrode PadSet was left on her chest in the HDU so that she could be monitored in the event she needed mechanical ventilatory support.

\section{Case 2}

A 21-y-old Kenyan man with a history of myasthenia gravis presented to Casualty with complaints of weakness that had progressively worsened over the previous week and that had resulted in endotracheal intubation during past hospitalizations. The patient was admitted to the HDU for observation and airway monitoring. During the evening, the patient became progressively weaker and was admitted to the ICU, where he was intubated.

Definitive therapy with intravenous immunoglobulin or plasmapheresis and maintenance therapy with pyridostigmine is the treatment of choice. ${ }^{7,8}$ However, intravenous immunoglobulin and plasmapheresis can be performed only in select tertiary care centers in Nairobi, and pyridostigmine was unavailable at the AIC Kijabe Hospital.

In the absence of pyridostigmine, the ICU team decided to use neostigmine to treat the myasthenic respiratory symptoms. A standard bolus injection dose of neostigmine is $0.5-2.5 \mathrm{mg}$ intravenously every $1-3 \mathrm{~h}$, yielding a range of $0.5-7.5 \mathrm{mg}$ intravenously every $3 \mathrm{~h} .{ }^{9}$ Although there are no data reporting the use neostigmine as an infusion for the treatment of myasthenic respiratory symptoms, regular bolus injection dosing was not considered to be reliable with the nursing infrastructure in the ICU at the AIC Kijabe Hospital. Therefore, the decision was made to deliver the neostigmine by continuous infusion.

The ICU team administered the neostigmine infusion at $1 \mathrm{mg} / \mathrm{h}$ with noninvasive $\dot{\mathrm{V}}_{\mathrm{E}}$ monitoring. The patient was placed on the RVM system with the $\dot{\mathrm{V}}_{\mathrm{E}}$ marker set at $80 \%$ predicted $\dot{\mathrm{V}}_{\mathrm{E}}(4.64 \mathrm{~L} / \mathrm{min})$ and the low $\dot{\mathrm{V}}_{\mathrm{E}}$ alarm set at $40 \%$ predicted $\dot{\mathrm{V}}_{\mathrm{E}}(2.32 \mathrm{~L} / \mathrm{min})$.

The patient was on pressure support spontaneous ventilation via a Puritan Bennett 840 ventilator. The amount of pressure support was set to deliver a $\dot{V}_{\mathrm{E}}$ of between $80 \%$ and $100 \%$ predicted $\dot{\mathrm{V}}_{\mathrm{E}}$ because the $\mathrm{V}_{\mathrm{T}}$ display on the ventilator available for the patient was not functioning. After starting the neostigmine infusion, the amount of pressure support was weaned without specific time constraints as long as the patient's $\dot{\mathrm{V}}_{\mathrm{E}}$ remained above $40 \%$ predicted $\dot{\mathrm{V}}_{\mathrm{E}}$. Within $8 \mathrm{~h}$, the patient was extubated and said he felt stronger than he had in weeks. RVM was used after extubation to monitor respiratory mechanics to ensure that the patient did not develop respiratory depression due to weakness, potentially leading to re-intubation.

The use of a neostigmine infusion with noninvasive $\dot{V}_{E}$ monitoring allowed the ICU team to safely extubate a patient with a profound neuromuscular disorder. RVM permitted ongoing respiratory assessment in the post-extubation period, providing the team with confidence that they would have an early warning of changes in respiratory status to enable timely intervention and prevent the requirement for emergent action following unappreciated deterioration.

\section{Case 3}

A 70-y-old Kenyan woman presented to Casualty after fevers, night sweats, and malaise for 2 weeks. Laboratory 


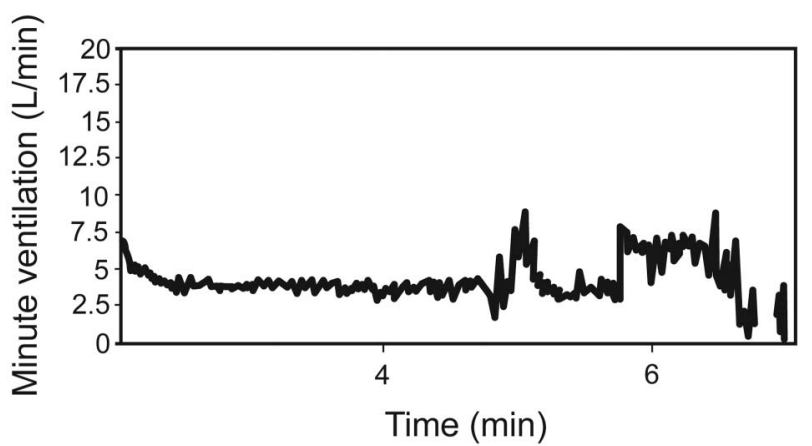

Fig. 2. Respiratory volume monitoring during withdrawal of care (Case 3) until death.

studies yielded a positive assay for Plasmodium falciparum malaria. She was started on antimalarial medications, but the hemolysis associated with P. falciparum had already started to damage her renal tubules, leading to acute kidney injury. ${ }^{10}$

The acute kidney injury progressed to increases in blood urea nitrogen and serum creatinine. With the increase in blood urea nitrogen, the patient became somnolent, and she was intubated for airway protection. The patient progressed to uremia and end-stage renal disease. Dialysis services are not available at the AIC Kijabe Hospital, and the disease had progressed to the point where renal transplantation would have been the only successful modality of treatment. The patient had subsequently developed and was being treated for ventilator-associated pneumonia.

Because of her comorbidities, the ICU team suggested that care should be withdrawn. Withdrawal of care straddles a fine line of providing patient comfort and committing euthanasia. Obviously, practitioners do not want to cause apnea with medication administration, but they do want to provide patient comfort to minimize patient and family anxiety.

Before extubation, $0.2 \mathrm{mg}$ of intravenous glycopyrrolate was administered to control oropharyngeal secretions. Fentanyl monotherapy was chosen for analgosedation during withdrawal of care. The percent-predicted $\dot{V}_{E}$ was programmed into the RVM while the patient was on pressure support spontaneous ventilation via the Puritan Bennett 840 ventilator. She was monitored using the RVM system with a $\dot{\mathrm{V}}_{\mathrm{E}}$ marker set at $80 \%$ predicted $\dot{\mathrm{V}}_{\mathrm{E}}(4.32 \mathrm{~L} / \mathrm{min})$ and the low $\dot{\mathrm{V}}_{\mathrm{E}}$ alarm set at $40 \%$ predicted $\dot{\mathrm{V}}_{\mathrm{E}}(2.16 \mathrm{~L} / \mathrm{min})$. The patient was extubated at 6:30 $\mathrm{AM}$, and fentanyl was given in 25- $\mu$ g aliquots until the $\dot{\mathrm{V}}_{\mathrm{E}}$ reached the $40 \%$ low $\dot{\mathrm{V}}_{\mathrm{E}}$ alarm. Once that threshold was reached, fentanyl administration was stopped. The $40 \%$ threshold was reached in $\sim 5$ min, and the patient died $\sim 15$ min later (Fig. 2). We quickly achieved patient comfort without committing euthanasia, and the family was at peace with the process.

\section{Discussion}

There are concerns when introducing new technologies in austere settings. The first major concern is cost. ${ }^{11} \mathrm{Al}-$ though the cost of a new RVM is approximately $\$ 5,000$, Respiratory Motion has permanently donated 2 monitors to the Kijabe Hospital under their global outreach program to improve patient care and education. The philanthropic mission is to improve patient care and practitioner education in the peri-intubation period and decrease the need for other expensive and invasive testing, such as arterial blood gas measurement. Even without donation, these monitors justify their expense through a reduction in laboratory cost and a potential improvement in patient safety and outcomes, as hypercarbia is usually underdiagnosed and is especially detrimental to the neurosurgical and heart failure patient population. ${ }^{12,13}$

The second major concern is maintenance. ${ }^{14}$ The RVM used works on a Microsoft (Redmond, Washington) Windows-based platform and provides an easy avenue for troubleshooting and fixing any malfunction. Additionally, technical support is available by telephone, and the practitioner may communicate via a wireless fidelity application without incurring telephone charges. All software upgrades can also be completed remotely; thus, there is no need for local biomedical engineering support.

Finally, the RVM does require disposable PadSets for the use of the product. The PadSets can be reused on multiple patients until the impedance disallows for accurate $\dot{\mathrm{V}}_{\mathrm{E}}$ monitoring. The PadSets are light, inexpensive, and easy to transport. Because there are several missionary groups traveling to Kijabe every year, the supply of PadSets can be replenished. Therefore, the major barriers to new technology in austere settings can be addressed.

Although 3 different cases were described, the RVM can also be used to assess airway obstruction. In a study examining 3 subjects during regular and obstructed breathing (by glottic closure), tracings from the RVM and a closed-cell spirometer (Morgan Scientific, Haverhill, Massachusetts) were correlated. Therefore, the RVM has the ability to detect airway obstruction with normal oxygenation before patient deterioration. ${ }^{2}$

The adoption of new technology is difficult. Patients were triaged regarding the need for peri-intubation monitoring when they were in a less monitored setting (transition from the ICU to HDU) and when clinical decision making would be aided by information other than that obtained from monitors, ventilators, and laboratory studies. Because RVM use is not yet widely accepted as best practice, clinical judgment was used to determine patient benefit and gather data to make informed decisions regarding the patient population that would derive the greatest benefit and the continued use of these monitors even when the practitioners in the ICU varied. 


\section{NoninVasive Respiratory Volume MONITORING}

Although RVM has been most widely used in postsurgical patients and during monitored anesthesia care, these 3 cases demonstrate other applications of the device. This real-time quantitative assessment of respiratory function has promise in both high-tech academic settings and austere environments.

\section{REFERENCES}

1. Voscopoulos C, Brayanov J, Ladd D, Lalli M, Panasyuk A, Freeman J. Special article: evaluation of a novel noninvasive respiration monitor providing continuous measurement of minute ventilation in ambulatory subjects in a variety of clinical scenarios. Anesth Analg 2013;117(1):91-100.

2. Panasyuk A, Lalli M, Panasyuk S, Yocum N, Desmarais L, Lew R, Freeman J. Assessment of a continuous monitoring technique to measure adequacy of respiration. Circulation 2011;124:A13398.

3. Voscopoulos CJ, MacNabb CM, Freeman J, Galvagno SM Jr, Ladd D, George E. Continuous noninvasive respiratory volume monitoring for the identification of patients at risk for opioid-induced respiratory depression and obstructive breathing patterns. J Trauma Acute Care Surg 2014;77(3 Suppl 2):S208-S215.

4. Voscopoulos C, Freeman J, Ladd D, Brayanov JB, MacNabb CM, George E. Use of a respiratory volume monitor to identify opioid induced respiratory depression in the post-anesthesia care unit. Anesth Analg 2013;116;S-1-S-390.

5. Voscopoulos C, MacNabb CM, Brayanov J, Freeman J, George E. Opioid-induced respiratory depression-evaluation of a new protocol to identify patients at risk. Anesthesiology 2013, October 12-16, 2013, San Francisco, CA. http://www.respiratorymotion.
com/storage/docs/RMI\%20Abstract\%20\%20ASA\%20MGH $\% 20$ 2013.pdf. Accessed January 5, 2015.

6. Young D, Harrison DA, Cuthbertson BH, Rowan K, TracMan C. Effect of early vs late tracheostomy placement on survival in patients receiving mechanical ventilation: the TracMan randomized trial. JAMA 2013;309(20):2121-2129.

7. Yu QS, Holloway HW, Luo W, Lahiri DK, Brossi A, Greig NH. Long-acting anticholinesterases for myasthenia gravis: synthesis and activities of quaternary phenylcarbamates of neostigmine, pyridostigmine, and physostigmine. Bioorg Med Chem 2010;18(13):4687-4693.

8. Barth D, Nabavi Nouri M, Ng E, Nwe P, Bril V. Comparison of IVIg and PLEX in patients with myasthenia gravis. Neurology 2011; 76(23):2017-2023

9. White L, Sandhu G. Continuous neostigmine infusion versus bolus neostigmine in refractory Ogilvie syndrome. Am J Emerg Med 2011; 29(5):576.e1-576.e3.

10. Saravu K, Rishikesh K, Parikh CR. Risk factors and outcomes stratified by severity of acute kidney injury in malaria. PLoS ONE 2014; 9(3):e90419.

11. Grimes CE, Henry JA, Maraka J, Mkandawire NC, Cotton M. Costeffectiveness of surgery in low- and middle-income countries: a systematic review. World J Surg 2014;38(1):252-263.

12. McGillicuddy JE, Kindt GW, Raisis JE, Miller CA. The relation of cerebral ischemia, hypoxia, and hypercarbia to the Cushing response. J Neurosurg 1978;48(5):730-740.

13. Viitanen A, Salmenperä M, Heinonen J. Right ventricular response to hypercarbia after cardiac surgery. Anesthesiology 1990;73(3):393400.

14. Dubowitz G, Detlefs S, McQueen KA. Global anesthesia workforce crisis: a preliminary survey revealing shortages contributing to undesirable outcomes and unsafe practices. World J Surg 2010;34(3): 438-444. 